\title{
Design and Evaluation of Robotic Steering of a Flexible Endoscope
}

\author{
Jeroen Ruiter, Esther Rozeboom, Mascha van der Voort, Maarten Bonnema, Ivo Broeders
}

\begin{abstract}
In current flexible endoscopy the physician faces problems in manipulating the endoscope. A lot of experience is required to master the procedure. This paper describes the design of an add-on robotic module that improves the user interface of traditional endoscopes and allows a single physician to operate it easily. We identified critical user aspects of traditional endoscopes that need to be copied in a robotic setup. In our design the physician uses a remote control that is connected to a light drive system. It allows manipulation of the robotic endoscope in space. We conducted an experiment to judge the usability of our system. Results indicate that robotic steering, using a position-controlled touchpad or a ratecontrolled joystick increases efficiency and satisfaction.
\end{abstract}

\section{INTRODUCTION}

$I_{\mathrm{g}}^{\mathrm{N}}$ flexible endoscopy the interior surfaces of the gastrointestinal, reproductive and respiratory tracts are assessed. The physician uses a flexible endoscope with a camera at the steerable distal tip that is introduced in the natural body openings. Experience is necessary to master the procedure of inserting, steering, and executing small interventions with a flexible endoscope. Performing a colonoscopy to inspect the large intestine is one of the most demanding diagnostic endoluminal procedures performed in the clinic (Fig. 1). According to [1]-[2] about 100 to 200 procedures are required to reach the level to perform the procedure safely and within reasonable time.

No revolutionary changes have occurred in endoscope technology during the last five decades. Main usability problems are related to the control section at the proximal end of the flexible endoscope. To obtain single person endoscope control, the control section is designed for the left hand, while the right hand manipulates the flexible shaft. Because of the configuration of control elements the physician often faces handling problems. For instance, approximately $20 \%$ of the physicians are using both hands for the control section, while an assistant manipulates the shaft according to spoken instructions [3]. This is undesirable since introducing the flexible endoscope into the tortuous and elastic colon is a delicate task that requires interpretation of force feedback information to support

Manuscript received January 31, 2012. This work was supported by the Dutch Ministry of Economic Affairs and the Province of Overijssel, within the Pieken in de Delta (PIDON) initiative.

Jeroen Ruiter (phone: +31(0)541 570 720, fax +31(0)541 570 721, j.g.ruiter@utwente.nl), Mascha van der Voort and Maarten Bonnema are with the Laboratory of Design, Production and Management, Department of Engineering Technology, University of Twente, Enschede, The Netherlands. Jeroen Ruiter is also with DEMCON advanced mechatronics, Oldenzaal, The Netherlands. Esther Rozeboom and Ivo Broeders are with the Laboratory of Minimal Invasive Surgery \& Robotics, Department of Technical Medicine, University of Twente, The Netherlands.

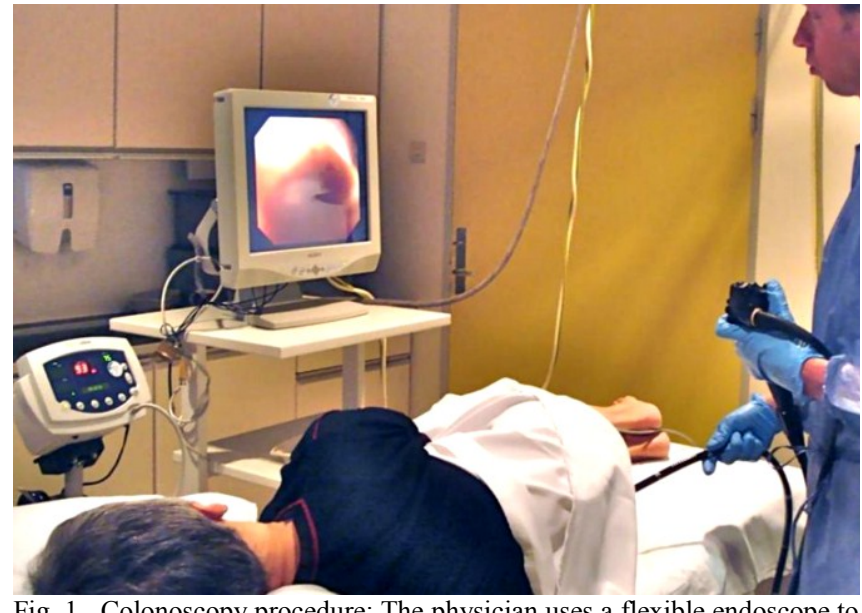

Fig. 1. Colonoscopy procedure: The physician uses a flexible endoscope to inspect the large intestine of a patient.

steering. Inefficient steering may lead to time loss and excessive stretching of the intestinal wall, leading to increased patient discomfort [4].

At present there are no flexible endoscopes available that can be controlled in an intuitive and user-friendly way by one person. A long term solution is to redesign the control section. However, despite the limited usability, current flexible endoscopes are widely used systems with qualities like cleanability, maneuverability, and good image quality [5]. We propose an add-on robotic module positioned on a traditional endoscope. The physician uses a remote control to actuate the bendable tip. Key factor is that tip steering and tip actuation are mechanically decoupled and computer intelligence is integrated. Robotic steering has the potential to improve usability, preserve current endoscope qualities, and prevent high costs related to replacement of endoscopic equipment. Acceptance is expected to be high since our robotic setup fits to the current workflow and infrastructure.

Allemann et al. [6] have developed a system with a game joystick to control a motorized traditional endoscope. In their evaluation both novices and experienced physicians required significantly more time to complete a given task when using a joystick compared to conventional controls. They concluded that possibly the limited maneuverability of the endoscope positioned in the setup is responsible for the disappointing results. Zhang et al. [7] performed a comparable experiment with a joystick controller and a motorized endoscope with a fixed position in the setup. They concluded that the time required to finish the process relies on the degree of familiarity with the robot system. After 3-5 test runs an expert in flexible endoscopy performs equal in both techniques. Nevertheless, the lack of proprioceptive 
feedback in robot supported manipulation was indicated to decrease the effectiveness of the system. Reilink et al. [8] conducted an experiment with a six degrees of freedom haptic controller, coupled to a stationary motorized traditional endoscope. Experts appeared faster when using the conventional steering method compared to motorized steering methods. Students who had done flexible endoscopy training showed no significant differences.

In all above work endoscope handling opportunities were inferior to the current manual steering design. We propose a hybrid setup in which mobile as well as stationary use of the robotic endoscope is possible. The control section of the robotic endoscope can be manipulated freely to resolve for instance shaft looping inside the lumen.

The experimental setup in the studies mentioned was not designed to be implemented in clinical practice. Our robotic endoscope is based on the clinical workflow and integrates medical and technical state of the art.

This paper discusses the design and evaluation of such a robotic flexible endoscope. In Section II shortcomings of the current user interface are discussed. Section III describes the design considerations for our system. In Section IV the design is specified and shown. Section V contains the results of the experiments that are conducted. Finally, Section VI concludes and provides directions for further work.

\section{CURRENT USER INTERFACE SHORTCOMINGS}

This section discusses the current problems related to physician-instrument interaction.

The left hand steers the distal tip by turning two navigation wheels on the control section (Fig. 2). The control of the tip orientation is not very intuitive because the navigation wheels are arranged in the same plane while the bendable portion will bend in two perpendicular directions. Single handed operation of the wheels is difficult due to size, position and force requirements, especially with small hands. In a survey of U.S. gastroenterology fellows, $41 \%$ of the respondents considered their hands too small for a standard endoscope's control section [9]. Some endoscopists release the grip of the right hand on the shaft and use it to turn the smaller outer wheel. The shaft position is maintained by trapping it between the physician's thigh and the examination table [10]. Other physicians use torque steering as an alternative technique. They turn the large navigation wheel, while the small wheel is locked in neutral position, and additionally torque the shaft of the endoscope [11].

The control section also contains manual operated valves to insufflate air or $\mathrm{CO} 2$ into the lumen, to rinse the camera lens, to suck fluids and gas out of the lumen, and some programmable switches for functions like taking a picture or switching to narrow band imaging. This complicates single handed operation even further [10].

As a consequence, manipulation of flexible endoscopes is associated with awkward body rotations, bending of the knees, and a variety of arm movements. These movements are in the clinic sometimes referred to as the endoscopy

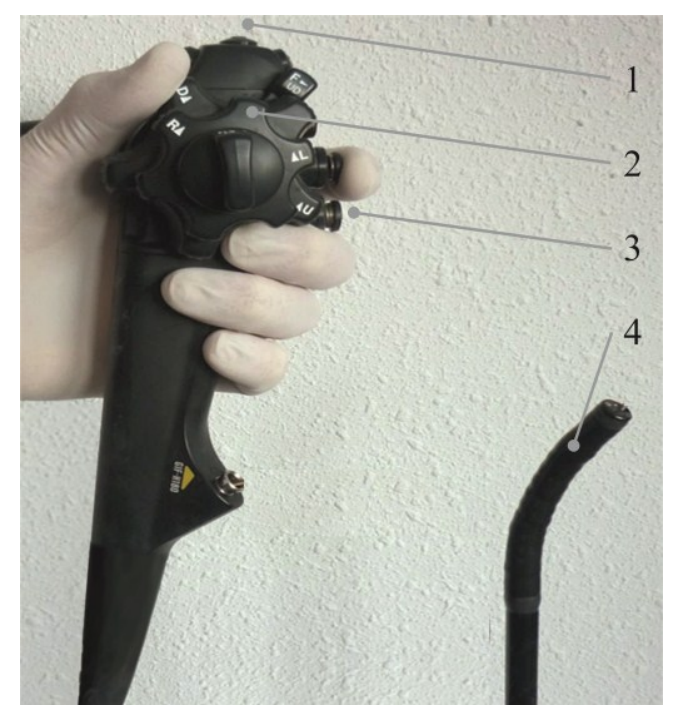

Fig. 2. Control section endoscope: (1) Programmable switches, (2) Navigation wheels, (3) Valves for insufflation, rinsing, and suction, (4) Steerable tip with camera.

dance and are especially performed during difficult parts of the procedure. The prevalence of musculoskeletal complaints has been shown to be higher for endoscopists than for other medical specialties [12]. Steering the navigation wheels and operating the control buttons of the control section require repetitive, extreme and prolonged wrist and finger flexion or extension. Manipulation of the shaft of a flexible endoscope is associated with awkward wrist, shoulder, and neck postures. In a questionnaire under colonoscopists concerning work related injuries, 226 out of the 608 respondents reported physical complaints obtained by performing colonoscopy. Most injuries were related to torquing the shaft and turning the dials [3].

It can thus be concluded that current endoscope handling is not ergonomic and user friendly. Physicians have learned to overcome the drawbacks, but at the expense of personal well-being.

\section{DESIGN DIRECTIONS FOR ROBOTIC STEERING}

In this section the opportunities for robotics for intuitive and user-friendly single person endoscope handling are discussed. Many alternative endoscopes have been developed to improve colonoscopy physically and technically for the operator and make it more comfortable for patients. However, none of them is commercial available. Gaglia et al. [13] highlight technical innovations of new endoscopic devices. All described systems are designed to be less skill dependent compared to the user interface of conventional endoscopes, but all of them are also based on a new design of the endoscope. As stated in the introduction, we believe that acceptance is higher if conventional endoscopes can be used.

The human-machine interface of the robotic endoscope should allow the physician to operate cooperatively with the robot. Thus, ergonomics and integration into the clinical workflow are essential elements of a successful design [14]. 


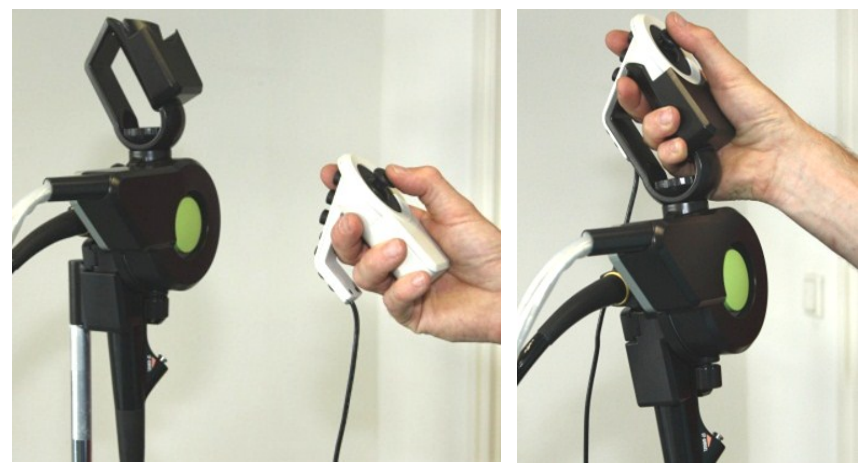

Fig. 3. Stationary (left) versus mobile use of the robotic endoscope

In the clinic the robotic module and the flexible endoscope are connected during preparation. The mechanical interfaces between the clean endoscope, the drive unit, and the user interface are critical. Even in case of non-sterile endoluminal interventions the patient should be protected against cross contamination. After the procedure, the assistant dismantles the robotic system for cleaning or replacement of disposable parts. The endoscope will be cleaned or disinfected according to the current clinical workflow.

Direct manipulation of the endoscope handle may be required to pass difficult parts of the lumen. We propose a hybrid setup that is configurable during the procedure. In one configuration the endoscope including the add-on robotic module is positioned in a docking station and the physician holds the remote control in one hand and the shaft in the other hand. In the other configuration the physician carries the robotic endoscope with the remote control that is directly coupled to the control section of the endoscope, as shown in Fig. 3. The former is easy to carry while the latter allows for extra maneuverability of the endoscope. Possibly this is beneficial during insertion of the endoscope. If necessary during a procedure the setup can also be changed to conventional steering by taking the endoscope in a few seconds out of the robot.

The remote control should be intuitive and suitable for single handed use. It ought to reduce musculoskeletal complaints of the operator and has to be operated close to the patient. All input controls of the control section of the current endoscope should be included in the remote control. During insertion the physician should be able to actuate insufflation, suction or rinsing while steering the tip. In current practice the flow is controlled by repeatedly actuation of the buttons (digital input). In the robotic setup flow could be controlled proportional (analog input). In particular precise (limited) insufflation contributes to successful insertion [15] and minimal patient discomfort [16]. The remote control can be operated while wearing disposable gloves and should allow for left as well as right handed use. The delicate task of shaft manipulation, that requires precise interpretation of force feedback information, can always be done with the dominant hand. This optimizes the setup for the about $10 \%$ left handed physicians [17].

Different kind of input controllers that are used for computer applications (e.g. mouse, joystick, haptic controller) are used for clinical systems as well. New innovations are related to speech, gaze, and gesture control. The clinical application of these innovative techniques is limited, mainly because of safety issues, like limited accuracy and robustness.

The input controller that steers the tip is a critical component with regard to usability and intuitive use. It should be able to manipulate the tip from $-180^{\circ}$ to $+180^{\circ}$ in left-right, up-down and combined (diagonal) directions. The controller should allow for fast large movements, precise small movements and stabilization of the bendable tip in a preferred position. For instance during inspection of the lumen, the tip should follow a smooth circular path. Thereby providing images of the entire mucosal surface within reasonable time and allowing for precise camera positioning to inspect suspicious areas.

In current practice the physician relates the forces required to turn the navigation wheels of a traditional endoscope to the shape of the tip and shaft inside the body. The navigation wheels transmit actuation forces to the tip by means of flexible Bowden cables. The force increases by friction in accordance with the degree of flexion of the endoscope. This helps the physician to estimate the flexion of the tip, interaction forces of the tip with tissue, and shaft loops that need to be straightened [18]. Force information from the navigation wheels need to be fed back to the physician in the robotic setup. Ideally this would be haptic feedback to achieve a sense of transparency but a haptic controller with at least 2 degrees of freedom that can be integrated with a small remote control is not available. Vision could provide an appropriate sensory substitute in the robotic setup. Indication bars reflecting force information are shown on the monitor. We have to verify in our setup if vision can adequately replace haptic information.

The setup limits the number of controllers suitable to steer the tip. A thumb joystick as seen in gamepad controllers or a touchpad like in laptops are the most likely options. Position and rate control are the two common transfer functions. In position control the input device indicates the desired position of the end effector, whereas in rate control the input device indicates the desired end effector velocity. There is no upfront evidence in literature to choose between these alternatives [19]. In our setup a thumb joystick combines best with rate control. It allows the physician to use the full bending range of the tip of the endoscope. Additionally, rate control can freeze the tip in a preferred position when releasing the joystick. The joystick, with spring loaded return-to-center functionality, returns to its initial position and sets the speed to zero. A touchpad can be best combined with incremental position control. Like in mouse navigation, clutching allows the physician to use the full manipulation range. Lifting the finger fixates the tip of the endoscope into position. In our experiment we try to determine the best control option.

Above considerations are implemented in our design of a robotic endoscope, as described in the next section. 


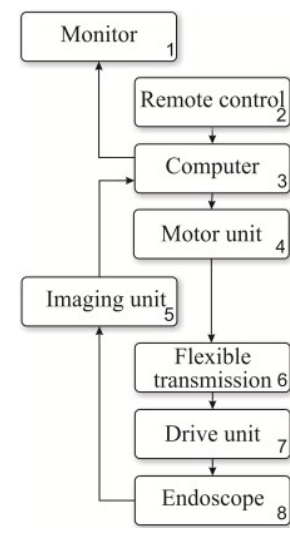

Fig. 4. System overview

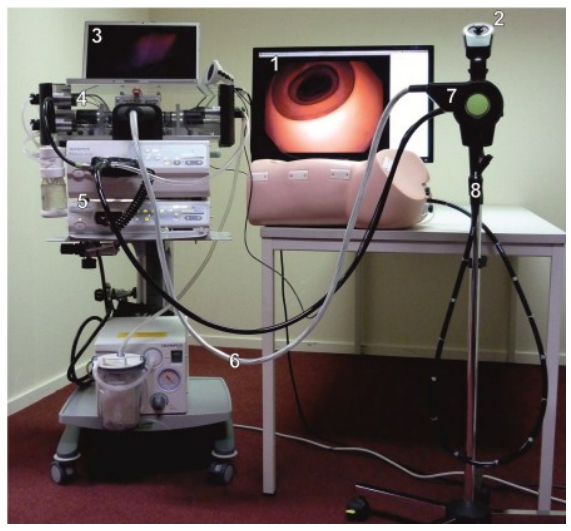

\section{DESIGN}

In Fig. 4 our system is depicted. The configuration is designed to obtain a light robotic endoscope that can be manipulated freely by the operator. For that reason all heavy components, like motors, are placed in a stationary positioned motor unit that is connected through a flexible transmission to a compact and light mobile drive unit. If the two motors for navigation wheel actuation would be positioned in the mobile drive unit it would add about $0.7 \mathrm{~kg}$ to the weight. The motor unit is placed on the endoscopy cart and the generic mobile drive unit connects with a dedicated interface to the navigation wheels of each individual type of endoscope. On top of the interface unit the holder of the remote control is positioned. Table 1 contains an overview of some general specifications of the designed mobile drive unit.

TABLE 1

SPECIFICATIONS OF THE DESIGNED MOBILE DRIVE UNIT

\begin{tabular}{ll}
\hline Maximum torque on wheels & $1.5 \mathrm{Nm}$ \\
\hline Range of motion & $360^{\circ}$ (omnidirectional) \\
\hline Maximum angular velocity & $2 \pi \mathrm{rad} / \mathrm{s}$ \\
\hline Weight mobile drive unit & $0.92 \mathrm{~kg}$ \\
\hline
\end{tabular}

\section{A. Drive system}

Antagonistic cable pairs between the stationary motor unit and the mobile drive unit actuate the navigation wheels of the endoscope (Fig. 5). The cables are pretensioned to prevent backlash and delay in control. The outer sheath of the Bowden cables at the load side are supported by load cells to measure the applied force to the navigation wheels. The drive system is self-locking so the position of the tip of the endoscope (camera position) is maintained when the controller of the remote control is not actuated. Two optical encoders are added to the load side to be able to improve control. In the current setup these are not in use.

Two DC servo motors were selected for actuation. The motors, motor controllers and power supply are all integrated in the motor unit box. The main program is computed on an external computer.

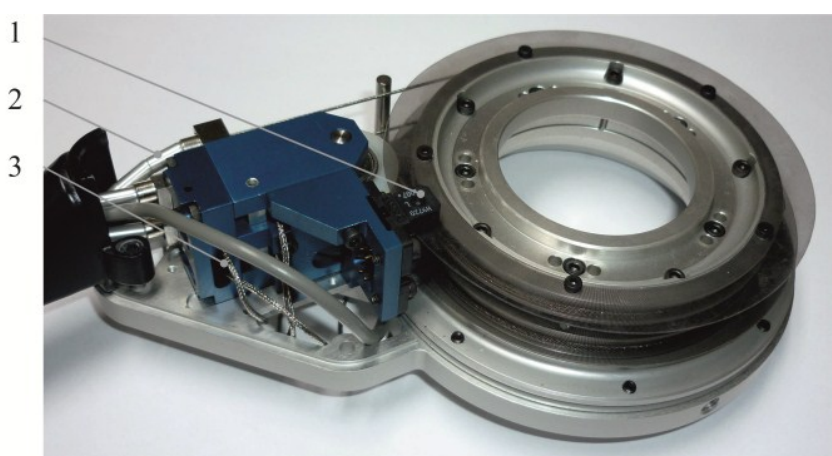

Fig. 5. Mobile drive unit: (1) Optical disc encoders, (2) Bowden cables, (3) Force sensors.

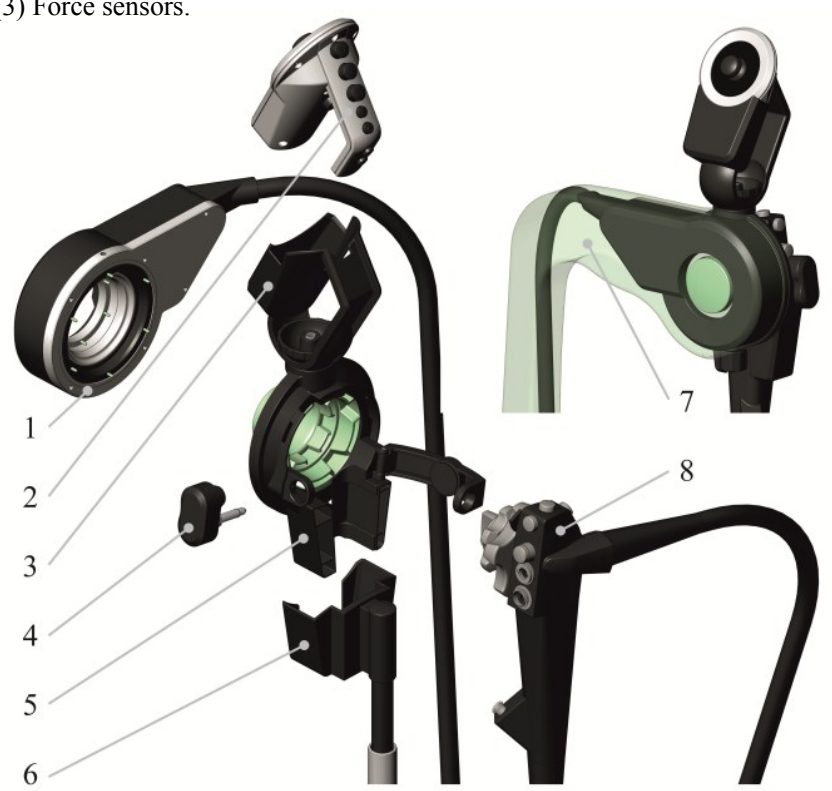

Fig. 6. Exploded view coupling mechanism: (1) Drive unit, (2) Remote control, (3) Holder remote control, (4) Plug, (5) Sterile interface, (6) Docking station, (7) Sterile bag, (8) Endoscope.

\section{B. Coupling mechanism}

The drive system cannot be sterilized. A sterile interface couples the drive unit to the clean endoscope to prevent cross contamination. If preferred the drive unit is sealed in a bag as shown in Fig. 6.

The interface is first connected to the endoscope. It is locked by an endoscope specific plug that bridges the valves of insufflation, rinsing, and suction on the endoscope so these can be controlled with the remote control. This assembly is subsequently connected with the mobile drive unit by threaded knobs. Torque between drive unit and interface is transferred with a pin hole connection.

A configurable holder for the remote control is integrated with the interface. The physician is able to position it to personal preferences to comfortably hold and carry the robotic endoscope. If preferred the robotic endoscope is positioned in a docking station on a pole cart and the remote control can be detached from the holder. The docking station allows axial rotation of the shaft of the endoscope that is induced by the physician during the procedure. 

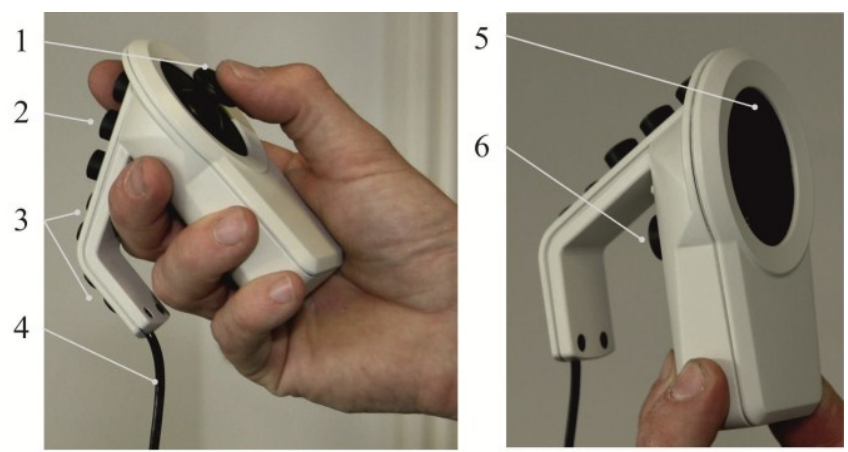

Fig. 7. Joystick and touchpad remote controllers: (1) Thumb joystick, (2) Buttons for insufflation, rinsing, and suction, (3) Programmable switches, (4) USB cable to computer, (5) Touchpad, (6) Hold-to-run safety switch.

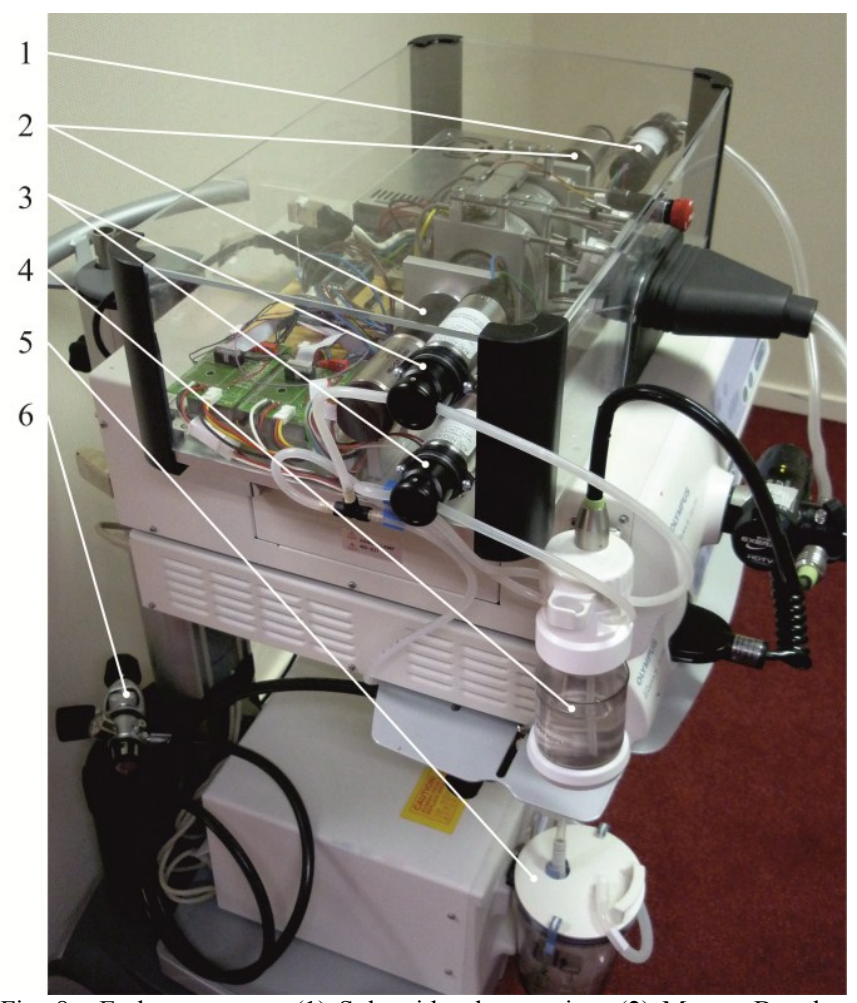

Fig. 8. Endoscopy cart: (1) Solenoid valve suction, (2) Motors Bowden cables, (3) Solenoid valves insufflation and rinsing, (4) Water container, (5) Pump unit, (6) Air/ $\mathrm{CO}_{2}$ gas cylinder.

\section{Remote control}

The remote control allows single handed control of all available functionality of a traditional endoscope, as shown in Fig. 7. A thumb joystick as well as a touchpad can be integrated as input controller to steer the tip. Push buttons are arranged to operate all valves and switches of the control section of the current endoscope. Buttons for proportional insufflation, rinsing, and suction are pressure sensitive by means of an underlying force sensing resistor. Flow is controlled with solenoid pinch valves that are positioned in the motor unit (Fig. 8). A hold-to-run safety switch needs to be pressed during operation of the robotic endoscope to prevent unintended actuation of input controls.

\section{Feedback information}

The operator is provided with several sources of visual

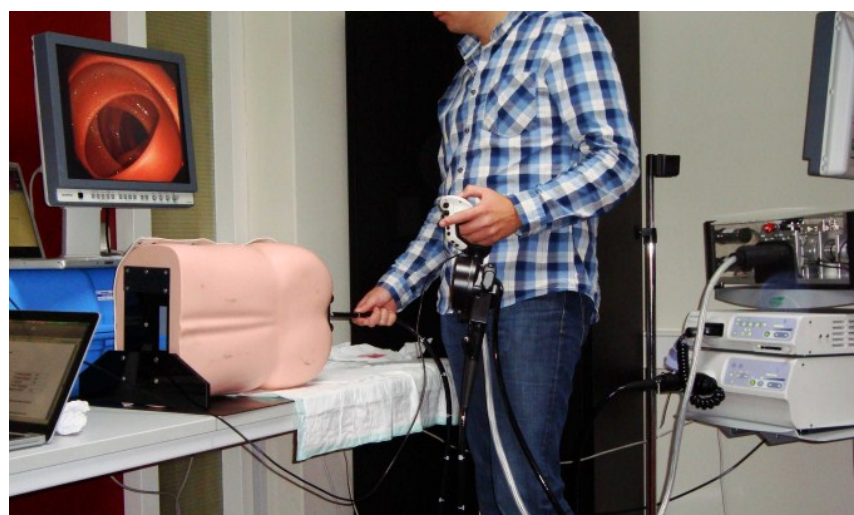

Fig. 9. Experimental setup

feedback to support control of the robotic endoscope. These are integrated in a single monitor (Fig. 4). The endoscopic images that visualize patient tissue are most important and take up most surface of the monitor. The endoscope and its imaging unit determine the characteristics of these images. Often these are provided in high definition.

One fourth of the monitor is reserved for additional feedback provided by the robotic system. The flexion of the endoscope tip is shown in a bending diagram. The diagram shows a bar in a white circle that extends from the center into the direction that the tip is moving. The direction and length of the bar are an indication for tip direction and flexion respectively.

Additional bar indicators provide information about the torque required to turn the navigation wheels. This relates to the shape of the shaft and interaction of the endoscope tip with tissue. Flow information of insufflation, rinsing, and suction is also fed back by bar indicators.

\section{EVALUATION}

\section{A. Experimental setup}

This section describes the experiments conducted to determine the optimal settings of our robotic endoscope and to assess its intuitiveness and user-friendliness. We compared conventional steering of the tip to robotic steering to obtain knowledge about the best input controller and the required maneuverability of the endoscope. The tested setups were:

1) Conventional steering with navigation wheels. We use this method as a reference for the robotic setups.

2) Joystick steering with stationary endoscope. The endoscope including the drive unit is positioned in a docking station and the subject only holds the remote control, as shown in Fig. 3.

3) Touchpad steering with stationary endoscope.

4) Joystick steering with mobile endoscope. The subject carries the endoscope with the remote control that is directly coupled to the control section of the endoscope, as shown in Fig. 3 and Fig. 9.

5) Touchpad steering with mobile endoscope.

A standard flexible colonoscope (Exeria II CF-H180AL, Olympus, Tokyo, Japan) and imaging unit (Exeria II CLV- 
TABLE 2

QUANTATIVE RESULTS EXPERIMENT

\begin{tabular}{|c|c|c|c|c|c|}
\hline Setup & $\begin{array}{c}\text { Conventional } \\
(\mathrm{n}=24)\end{array}$ & $\begin{array}{c}\text { Joystick - stationary } \\
\text { scope }(n=12)\end{array}$ & $\begin{array}{c}\text { Touchpad - stationary } \\
\text { scope }(n=12)\end{array}$ & $\begin{array}{c}\text { Joystick - } \\
\text { mobile scope }(n=12)\end{array}$ & $\begin{array}{c}\text { Touchpad - } \\
\text { mobile scope }(n=12)\end{array}$ \\
\hline${\text { Insertion time }(\mathrm{s})^{\mathrm{a}}}$ & $178(75)$ & $145(50)$ & $188(94)$ & $150(70)$ & $108(68)$ \\
\hline Workload $(\max .25)^{\mathrm{a}}$ & $16(4)$ & $13(4)$ & $10(4)$ & $11(4)$ & $15(4)$ \\
\hline Detection rate $(\%)^{\mathrm{a}}$ & $68(16)$ & $86(22)$ & $71(22)$ & $64(26)$ & $71(19)$ \\
\hline
\end{tabular}

180, Olympus, Tokyo, Japan) were used for all experimental conditions.

Subjects, without experience in handling an endoscope and without medical background, were asked to perform 2 colonoscopy tasks on an anatomical model (M40, Kyoto Kagaku, Kyoto, Japan). The absence of experience enabled testing of intuitiveness. First, subjects had to advance and steer the endoscope up to the point where the colon starts, the cecum. Secondly the endoscope had to be retracted to the rectum while inspecting the mucosal surfaces for lesions, represented by 7 prepositioned red blocks sized $2 \times 2 \times 1 \mathrm{~mm}$.

It was too time consuming to test all setups on all individual participants. For that reason the population of 24 subjects (aged 21-50 years, 7 women and 17 men) was divided over 2 groups. One group tested setup 1, 2 and 5 . The other group tested setup 1, 3 and 4 . This way all participants experienced both input controllers and both settings of endoscope handling. Each of the six possible orders of the three conditions was performed equally often to correct for learning effects and fatigue. For each setup 5 minutes of practice time was available and the opportunity to ask for advice on usage.

An easy bowel configuration was chosen in which all subjects could complete the task. Our focus was to test the steering usability of the endoscope. Future experiments by experienced physicians will be more challenging to test also the (clinical) usability of features like insufflation, suction and force feedback information that are typically required in difficult procedures. These functionalities were not available in this novices experiment.

Usability is defined by the International Standardisation Organisation (ISO) as: "the extent to which a product can be used by specific users to achieve goals with effectiveness, efficiency, and satisfaction in a specified context of use" [20]. The three factors are widely accepted to concern distinct measures and were evaluated in the experiment [21]. In our experiment the following dependent variables were measured:

- Full insertion into the cecum (effectiveness)

- Detection rate of lesions (effectiveness)

- Insertion time (efficiency)

- Subjective workload analysis measuring mental and physical demand, performance, effort and frustration. Based on assigning scores to a modified NASA Task Load Index, [22] (efficiency)

- Rank interfaces according to preference (satisfaction)

- Questionnaire by interview (satisfaction)

\section{B. Results and discussion}

The quantitative results of the experiment are depicted in Table 2 . The results indicate that robotic steering by novices improves insertion time and workload experience. Compared to the conventional method, joystick steering with stationary endoscope and touchpad steering with mobile endoscope had significantly faster insertion times. The workload scoring of all robotic setups are significantly better than the conventional method, except for touchpad steering with mobile endoscope. The detection rate was not significantly affected by robotic steering.

All subjects performed full insertion into the cecum. Extensive maneuvering of the endoscope shaft was not required during insertion. For that reason nothing conclusive can be said on the necessity of a mobile scope. Future experiments with physicians in a challenging procedure should provide more knowledge. In the interviews all participants complain about the additional weight that needs to be carried in the mobile endoscope setup. One might consider free manipulation only in awkward circumstances such as looping of the shaft. During easy parts of the procedure the scope is docked. Despite the additional weight, novices appreciated the ergonomics and work posture of all robotic setups more than of the conventional setup.

The conventional steering method would be the first choice for one subject, and last in 16 of 24 subjects. Subjects picked the joystick control (mobile and stationary) first in 15 of 24 cases. Although subjects prefer joystick to touchpad control, data on performance does not endorse that outcome. Possibly, the reduced proprioceptive feedback in touchpad control limits the feeling of being in control. In addition, subjects tend to roll the thumb during touchpad control instead of only moving the tip of the thumb over the touch surface. In this case, tip movement will not occur as expected, since the center of the touched surface is not moved as intended. In the mobile setup, rolling the thumb is restricted by the additional weight and the imposed position of the hand with respect to the endoscope, possibly explaining the faster insertion time of the mobile compared to the stationary setup. A system that encourages steering with the tip of the thumb will likely lead to improved touchpad control.

Almost all participants thought that the joystick as well as the touchpad controller were too sensitive, but they valued the intuitiveness of operation and experienced no delay in tip response. Previous experience with game controllers or touch interfaces did not influence the outcome. Some 
suggested that up-down in joystick control should be reversed to copy flight control. Sensitivity as well as updown direction could be made adaptable to comply with user preferences.

Force feedback information from the navigation wheels was not available in the robotic setup in our experiment. However, the bending diagram, as described in Section IV.D, was shown during the experiment. We estimated that feedback on the extent to which the tip is bent was essential even in an easy bowel configuration. Novices appreciated this substitute for haptic feedback very much.

\section{CONCLUSION AND FUTURE WORK}

A robotic system is built that allows ergonomic single person control while preserving current endoscope qualities. Acceptance is expected to be high since our robotic setup can easily be implemented in the current clinical workflow. We showed that robotic steering by novices, using touchpad or joystick control, increases efficiency and satisfaction. The effectiveness was not significantly affected by robotic steering. Our results did not show a clear preference for a position-controlled touchpad or a rate-controlled joystick. We will perform additional experiments in which we will critically look at the type of input controller and the accompanying control algorithm.

Breaking the mechanical linkage and integrating computer intelligence between operator and end effector provides opportunities for improved usability. However, we also identified critical user aspects of traditional flexible endoscopes that are related to mechanical interfaces, like force feedback. These are probably required in a robotic setup, especially in challenging procedures. We will work on optimizing functionality proposed in Section IV that was not yet implemented (e.g. insufflation) or facilities that could not be well tested in this experimental setup (e.g. mobile versus stationary endoscope). Expert testing is required to test performance in clinically relevant advanced procedures.

\section{REFERENCES}

[1] P. S. Tassios, S. D. Ladas, I. Grammenos, K. Demertzis, and S. A. Raptis, "Acquisition of competence in colonoscopy: the learning curve of trainees," Endoscopy, vol. 31, pp. 702-706, Nov. 1999.

[2] G. C. Harewood, "Relationship of colonoscopy completion rates and endoscopist features," Digestive Diseases and Sciences, vol. 50, pp. 47-51, Jan. 2005.

[3] A. S. Liberman, I. Shrier, and P. H. Gordon, "Injuries sustained by colorectal surgeons performing colonoscopy," Surgical endoscopy, vol. 19, pp. 1606-1609, Oct. 2005.

[4] W. H. Kim, Y. J Cho, J. Y. Park, P. K. Min, J. K. Kang, and I. S. Park, "Factors affecting insertion time and patient discomfort during colonoscopy," Gastrointestinal Endoscopy, vol. 52, pp. 600-605, June 2000 .

[5] B. Bardou, F. Nageotte, P. Zanne, and M. de Mathelin, "Design of a telemanipulated system for transluminal surgery," in Proc. of 31st Annual International Conference of the IEEE EMBS, Minnesota, USA, 2009, pp. 5577-5582.

[6] P. Allemann, L. Ott, M. Asakuma, N. Masson, S. Perretta, B. Dallemagne, D. Coumaros, M. De Mathelin, L. Soler, and J. Marescaux, "Joystick interfaces are not suitable for robotized endoscope applied to NOTES," Surgical Innovation, vol. 16, pp. 111116, June 2009.
[7] S. H. Zhang, D. X. Wang, Y. R. Zhang, Y. H. Wang, Y.G. Wang, X.P. $\mathrm{Ma}$, "The human machine interface implementation for the robot assisted endoscopic surgery system. In Proc. of the International Workshop on Robot and Human Interactive Communication, Berlin, Germany, 2002, pp. 442-447.

[8] R. Reilink, S. Stramigioli, A. M. L. Kappers, and S. Misra, "Evaluation of flexible endoscope steering using haptic guidance, The International Journal of Medical Robotics and Computer Assisted Surgery, vol. 7, pp. 178-186, June 2011.

[9] D. Cohen, J. R. Naik, L. J. Tamariz, R. D. Madanick, "The perception of gastroenterology fellows towards the relationship between hand size and endoscopic training," Digestive Diseases and Sciences, vol. 53, pp. 1902-1909, July 2008.

[10] C. Williams, "Insertion technique," In Colonoscopy: Principles and Practice, 2nd ed., J. D. Waye, D. K. Rex, and C.B. Williams, 2nd edition, Chichester: Wiley-Blackwell, 2009, pp. 537-559.

[11] A. K. Shergill, K. R. McQuaid, and D. Rempel, "Ergonomics and GI endoscopy," Gastrointestinal Endoscopy, vol. 70, pp. 145-153, July 2009.

[12] S. L. Hansel, M. D. Crowell, D. S. Pardi, E. P. Bouras, and J. K. DiBaise, "Prevalence and impact of musculoskeletal injury among endoscopists: a controlled pilot study," Journal of Clinical Gastroenterology, vol. 43, pp. 399-404, May 2009.

[13] A. Gaglia, I. S. Papanikolaou, and W. Veltzke-Schlieker, "New endoscopy devices to improve population adherence to colorectal cancer prevention programs," World Journal of Gastrointestinal Endoscopy, vol. 2, pp. 244-251, July 2010.

[14] G. D. Hager, A. M. Okamura, P. Kazanzides, L. L. Whitcomb, G. Fichtinger, and R. H. Taylor, "Surgical and interventional robotics: Surgical assistance systems," IEEE Robotics and Automation Magazine, vol. 15, pp. 84-93, Dec. 2008.

[15] I. C. Roberts-Thomson and Edward Teo, "Colonoscopy: Art or science?," Journal of Gastroenterology and Hepatology, vol. 24, pp. 800-804, Feb. 2009.

[16] M. Bretthauer, G. S. Hoff, E. Thiis-Evensen, G. Huppertz-Hauss, and E. Skovlund, "Air and carbon dioxide volumes insufflated during colonoscopy," Gastrointestinal Endoscopy, vol. 58, pp. 203-206, Aug. 2003.

[17] C. Hardyck and L. F. Petrinovich, "Left handedness," Psychological Bulletin, vol. 84, pp. 385-404, May 1977.

[18] O. Körner and R. Männer, "Implementation of a haptic interface for a virtual reality simulator for flexible endoscopy," in Proc. of 11th International Symposium on Haptic Interfaces for Virtual Environment and Teleoperator Systems, Los Angeles, USA, 2003, pp. 278-284.

[19] S. Zhai, "Human performance in six degree of freedom input control," Ph.D. dissertation, Dept. of Industrial Engineering, University of Toronto, 1995.

[20] Ergonomic requirements for office work with visual display terminals (VDTs) - Part 11: Guidance on usability, ISO 9241-11:1998(E).

[21] K. Hornbæk, "Current practice in measuring usability: Challenges to usability studies and research," International Journal of HumanComputer Studies, vol. 64, pp. 79-102, Feb. 2006.

[22] S. G. Hart and L. E. Staveland, "Development of NASA-TLX (Task Load Index): Results of empirical and theoretical research," In Human Mental Workload, P. A. Hancock and N. Meshkati, Amsterdam: North Holland Press, 1988, 239-250. 